\title{
1 Non-adherence to thromboprophylaxis guidelines in atrial fibrillation: A narrative \\ 2 review of the extent and factors for guideline non-adherence
}

\section{Running heading: Thromboprophylaxis guideline non-adherence in atrial fibrillation}

4 Eyob Alemayehu Gebreyohannes ${ }^{1 *}$, Sandra Salter $^{1}$, Leanne Chalmers ${ }^{2}$, Luke Bereznicki ${ }^{3}$, Kenneth Lee ${ }^{1,3}$

$5 \quad{ }^{1}$ Division of Pharmacy, School of Allied Health, University of Western Australia, WA, Australia

$6 \quad{ }^{2}$ School of Pharmacy and Biomedical Sciences, Curtin University, WA, Australia

$7 \quad{ }^{3}$ School of Pharmacy and Pharmacology, University of Tasmania, TAS, Australia

$8 \quad$ Corresponding Author:

9 Email: eyob.gebreyohannes@ research.uwa.edu.au; justeyob@gmail.com

ORCID: 0000-0002-0075-4553

\section{Acknowledgements}

Mr. Eyob Alemayehu Gebreyohannes is a Ph.D. student and a recipient of the University of Western Australia International Fee Scholarship and University Postgraduate Award. Mr. Gebreyohannes would like to acknowledge the University of Western Australia for supporting his studies.

\section{Abstract}

Atrial fibrillation is the most common arrhythmia. It increases the risk of thromboembolism by up to 5-fold. Guidelines provide evidence-based recommendations to effectively mitigate thromboembolic events using oral anticoagulants (OACs) while minimizing the risk of bleeding. This review focuses on non-adherence to contemporary guidelines and the factors associated with guideline non-adherence. The extent of guideline nonadherence is different based on geographic region, the healthcare setting, and the risk stratification tools used. There has been a gradual improvement in guideline adherence over recent years, but a significant proportion of patients are still not receiving guideline-recommended therapy. Physician-related and patient-related factors (such as patient refusals, bleeding risk, older age, and recurrent falls) also contribute to guideline non-adherence, especially to undertreatment. Quality improvement initiatives that focus on undertreatment especially in the primary healthcare setting may help to improve guideline adherence. 


\section{Key points}

- A significant proportion of patients with atrial fibrillation across a range of health settings are still receiving guideline non-adherent anticoagulation, the extent of which is highly variable.

- Physician-related (such as a greater emphasis on the risk of bleeding than the risk of stroke) and patientrelated (such as patient refusals, bleeding risk, older age, and recurrent falls) factors contribute to guideline non-adherence.

- Guideline non-adherence is primarily because of undertreatment and appears to be higher in high-risk AF patients attending primary healthcare than for patients attending hospitals and cardiology practices. Quality improvement initiatives should focus on addressing undertreatment, especially in the primary healthcare setting.

\section{Introduction}

Atrial fibrillation (AF) is the most commonly encountered arrhythmia in clinical practice, affecting 37.5 million people around the world in 2017. [1] AF increases the risk of stroke and systemic embolism by up to 5-fold. [2] It is the primary cause of cardioembolic stroke and results in a more severe type of stroke with a higher mortality rate when compared to strokes in people without AF. [2,3] The past two decades saw a 33\% increase in the prevalence of AF which is expected to continue increasing in the coming three decades. [1] With increasing incidence and prevalence rates, AF poses a substantial burden in terms of the rate of hospitalization, morbidity, and mortality. [4, 5]

Oral anticoagulants (OACs) such as warfarin have proven effectiveness in reducing the incidence of stroke and systemic embolism in patients with AF. [6, 7] As such, they are one of the cornerstones of AF management. [5] In the past decade, the development of direct oral anticoagulants (DOACs) increased anticoagulation options available for use in AF. [8] Their convenience and better safety profile compared to the vitamin $\mathrm{K}$ antagonist (VKA), warfarin, has led to a decrease in the utilization of the latter and a rise in the utilization of these newer agents. However, warfarin is still prescribed for many patients with AF. [9]

Since 2013, seven evidence-based guidelines have been recently published by different cardiology expert groups that include sections to guide the utilization of anticoagulants in stroke and systemic embolism prevention in AF. $[5,10-16]$ 
53 Not all patients with AF have an identical risk of stroke. One of the primary determinants of the risk of stroke is

54 the type of AF. [5, 14] Traditionally, AF has been categorized as either valvular or non-valvular (NVAF). [17]

55 The majority of guidelines define valvular AF as the presence of moderate-to-severe mitral stenosis and/or a mechanical heart valve prosthesis. [5, 10, 13-15] Currently, only VKAs are recommended for the prevention of

57 stroke in patients with valvular AF. [5, 10, 13, 14] The CHEST AF guideline [12] uses the terms "a functional Evaluated Heart valves, Rheumatic or Artificial (EHRA)" type 1 or type 2 in place of valvular AF and NVAF, respectively; although these differences in terminology have no impact on clinical decision-making in these patients $[5,10,13,14]$.

61 Anticoagulation in patients with NVAF requires estimating the risk of stroke and selecting an appropriate anticoagulant from a number of options. [5, 7, 10-16, 18] Most guidelines [10, 12-14, 16] recommend the $\mathrm{CHA}_{2} \mathrm{DS}_{2}$-VASc score (congestive heart failure, hypertension, age $\geq 75$ (doubled), diabetes, stroke (doubled), vascular disease, age 65-74, and female sex category) as a means of stratifying patients' stroke risk (Figure 1). To avoid using different scores in men and women, the Australian AF guideline [5] recommends using the $\mathrm{CHA}_{2} \mathrm{DS}_{2}$ VA score, i.e. $\mathrm{CHA}_{2} \mathrm{DS}_{2}$-VASc without the sex category. On the other hand, the 2018 CHEST guideline [12] recommends that the $\mathrm{CHA}_{2} \mathrm{DS}_{2}$-VASc score be used only to initially identify patients who are at low risk. Anticoagulation should be provided to patients other than those who are at low risk. 


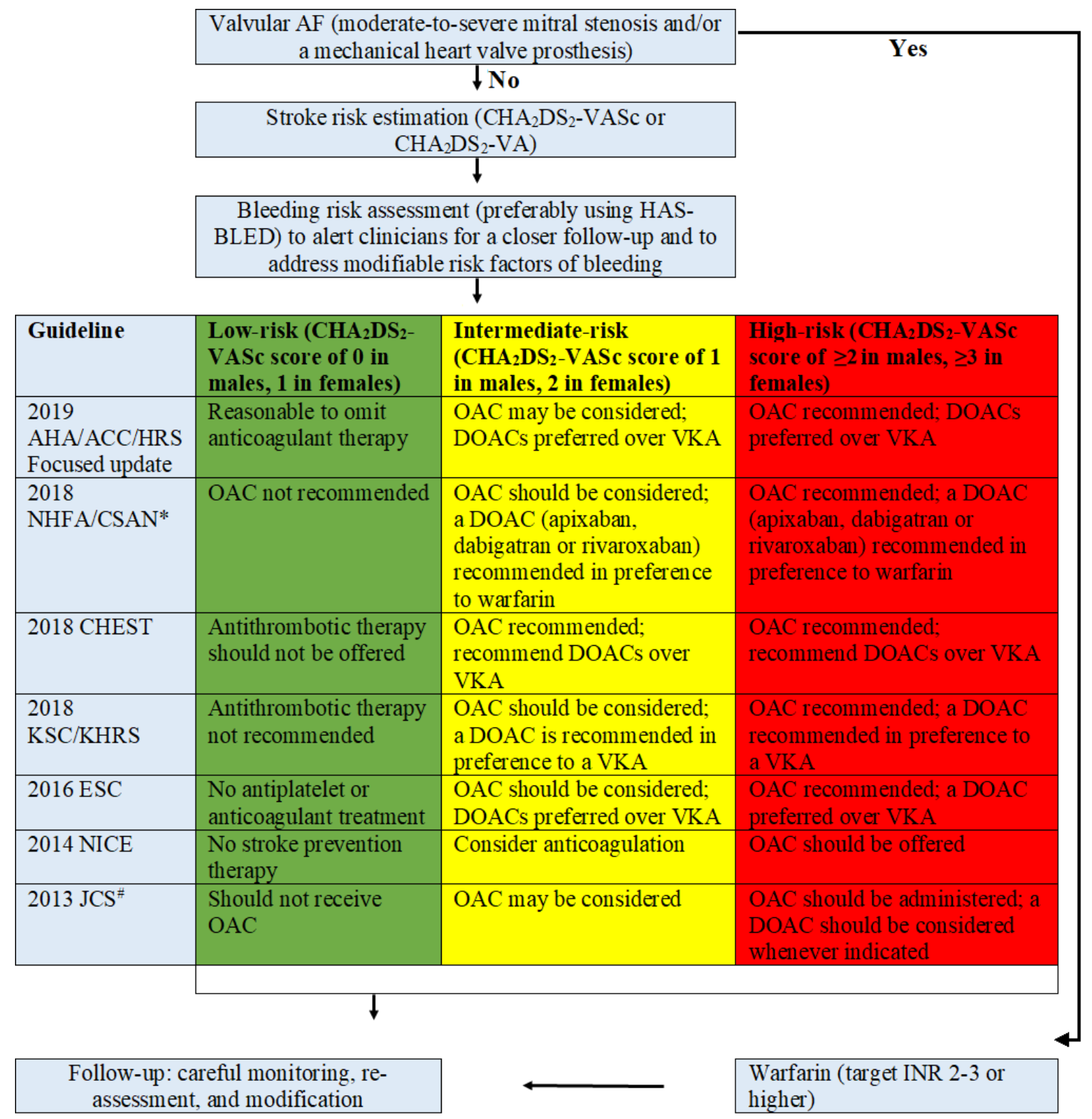

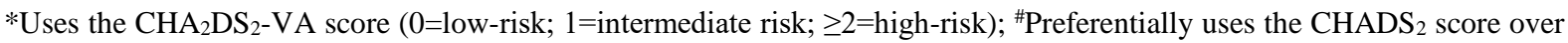
the $\mathrm{CHA}_{2} \mathrm{DS}_{2}$-VASc score; AHA/ACC/HRS: American Heart Association/American College of Cardiology/Heart Rhythm Society; $\mathrm{CHA}_{2} \mathrm{DS}_{2}$-VASc score: congestive heart failure, hypertension, age $\geq 75$ (doubled), diabetes, stroke (doubled)-vascular disease, age 65-74, and sex category (female); JCS: Japanese Circulation Society; KSC/KHRS: Korean Society of Cardiology/Korean Heart Rhythm Society; NHFA/CSAN: National Heart Foundation of Australia/Cardiac Society of Australia and New Zealand.

Figure 1: A summary of guideline recommendations on thromboprophylaxis in patients with AF

Similar to the stroke risk, patients' risk of bleeding should also be assessed in patients for whom anticoagulation is being considered. Various bleeding risk assessment tools including HAS-BLED, ATRIA, ORBIT, HEMOR$\mathrm{R}_{2} \mathrm{HAGES}$, GARFIELD, and the ABC are available. Of these, the HAS-BLED score (hypertension, abnormal renal/liver function (1 point each), stroke, bleeding history or predisposition, labile INR, elderly (>65), drugs/alcohol concomitantly (1 point each)) is recommended by most guidelines. $[10,12,13,15,16]$ It should be 
noted that the risk of bleeding increases with an increasing risk of stroke. [5, 14] Therefore, the purpose of bleeding risk assessment is to identify patients in whom OACs must be used with caution, and to identify and address modifiable risk factors for bleeding, not to withhold anticoagulation in patients who need it. [5, 12-14, 16] The guidelines also stress the need for due attention and adjustment as necessary on each follow up considering the dynamic natures of bleeding and stroke risks. $[12,13]$

Guideline non-adherence is associated with an increase in thromboembolic events because of undertreatment or, may increase the risk of bleeding including intracranial bleeding because of overtreatment. [18, 19-22] On the other hand, the benefit of guideline adherence in improving patient outcomes has been supported by the literature. [23] Evidence shows that non-adherence to evidence-based guideline recommendations and risk stratification tools for $\mathrm{AF}$ is a common occurrence even with the availability of DOACs. [24-26] Several reviews on thromboprophylaxis guideline non-adherence in patients with AF have been published in recent years. Two studies from 2015 focused only on undertreatment [27] and DOACs [23]. One study included only studies that utilized registry data [23]. A 2018 study reviewed randomized clinical trials and real-life outcomes and focused on DOACs. [28] A further review focused on trends in the prescription of antithrombotic medications, and patient compliance and persistence to OACs. [29] The present review seeks to determine the extent of non-adherence to thromboprophylaxis guidelines in AF and factors associated with guideline non-adherence. To ensure that this review is applicable to contemporary clinical practice, we focused on studies published in the past five years.

\section{Methods}

This narrative review is based on a key word search conducted in the PubMed and the Cumulative Index of Nursing and Allied Health Literature (CINAHL) Plus databases. The following search string was utilized to retrieve articles published until 5 May 2020: “atrial fibrillation” AND (anticoagul* OR antithrombo* OR anticoagul* OR anti-thrombo* OR "stroke prophyl*" OR "stroke prevention" OR thromboprophyl*) AND ("guideline adherence"). Studies that assessed non-adherence to one or more thromboprophylaxis guidelines, and studies that reported factors associated with guideline non-adherence were included. Both qualitative and quantitative studies published in English since 2015 were included. We begin our discussion with reported extent of guideline nonadherence by focusing mainly on undertreatment. This will be followed by trends in the extent of guideline nonadherence and studies that compared differences in guideline non-adherence across multiple geographic regions. Then, the extent of guideline non-adherence will be discussed by geographically categorizing studies that used 
the $\mathrm{CHA}_{2} \mathrm{DS}_{2}$-VASc score and were published in the past five years. Lastly factors affecting guideline nonadherence will be discussed.

\section{Non-adherence to guidelines}

Use of OACs for thromboprophylaxis in patients with AF in adherence to the evidence-based recommendations incorporated into clinical guidelines (i.e. 'guideline adherence') improve patient outcomes; conversely, patients whose therapy is non-adherent to guidelines experience poorer outcomes. [18, 19-22] Therefore, there has been a significant focus in the literature on quantifying guideline adherence in this clinical setting. In this section, the overall extent of guideline non-adherence is described. In addition, changes over time, and differences in guideline non-adherence in different areas of the world and in different healthcare settings are explored.

Reported rates of guideline non-adherence vary widely depending on the type of guideline and/or risk stratification tool used, the region, the study setting, and the specific group of patients included. Undertreatment is typically defined as no OAC treatment in patients at high risk of stroke such as those with valvular AF, or those with NVAF and a $\mathrm{CHA}_{2} \mathrm{DS}_{2}$-VASc score of $\geq 2$ in males ( $\geq 3$ in females), or a $\mathrm{CHADS}_{2} / \mathrm{CHA}_{2} \mathrm{DS}_{2}-\mathrm{VA}$ score of $\geq 2$. [5, 10, 1216] Overtreatment is defined as the use of antithrombotic agents in patients with AF who have no additional risk factors for thromboembolism and without any other indication. In particular, administration of any antithrombotic agent for thromboprophylaxis in patients with $\mathrm{NVAF}$ and a $\mathrm{CHA}_{2} \mathrm{DS}_{2}-\mathrm{VASc}$ score of 0 in males (1 in females) or a $\mathrm{CHADS}_{2} / \mathrm{CHA}_{2} \mathrm{DS}_{2}$-VA score of 0 is considered overtreatment, as no contemporary guidelines recommend therapy in these patients. $[5,10,12-16]$ The management of NVAF patients with a $\mathrm{CHA}_{2} \mathrm{DS}_{2}-\mathrm{VASc}$ score of 1 in males ( 2 in females) depends on the guideline used.

Non-adherence to guideline recommendations was observed in $4.4 \%$ to $95.2 \%$ of patients in the studies included in this review. The proportion of patients undertreated ranged from $2.5 \%$ [30] to $76.3 \%$ [31]. To clearly identify guideline non-adherence, undertreatment in particular, several studies included only patients at high risk for stroke. [32-42] The patients included in these studies either had $\mathrm{CHA}_{2} \mathrm{DS}_{2}-\mathrm{VASc}$ or $\mathrm{CHADS}_{2}$ scores $\geq 2$ [33-40] or were aged $\geq 75$ years old $[32,41,42]$, all of which made them at high risk. Undertreatment in these high-risk patients ranged from as low as $19.7 \%$ [38] to as high as $95.2 \%$ [40], with the majority of the studies reporting undertreatment between $40 \%$ and 50\%. [32, 33, 35-37, 39, 42] Of note, the study by Piccini et al., with $19.7 \%$ undertreatment, was part of a quality improvement initiative in hospitalized patients targeting guideline adherence and the extent of undertreatment before hospital admission, and before the intervention, in this study was $40.5 \%$. [38] In the study by Formiga et al., the relatively higher proportion of octogenarians (40\%) may have been the 

adherence over time. Cowan et al. [63] reported an increase in the prescription of OACs (by 30.6\%) and a decline in the prescription of antiplatelet agents (by 26.8\%) over a 10-year period in AF patients at high risk of stroke. Non-prescription of OACs in AF patients at a significant risk of stroke is declining. [33, 37, 64] While the prescription of antithrombotic agents in truly low-risk patients may be decreasing in some areas [64], an increasing trend has been reported in others. For instance, a 2020 Japanese study [65] reported that prescription of OACs in patients with a $\mathrm{CHADS}_{2}$ score of 0 increased from $53 \%$ to $66 \%$. However, one should note that the Japanese AF guideline [15] also recommends OACs in patients with a $\mathrm{CHADS}_{2}$ score of 0 but with additional risk factors for thromboembolism such as cardiomyopathy, vascular disease, and age between 65 and 74 years. This may be one potential reason for overtreatment in some of these patients despite their low risk of stroke as per the $\mathrm{CHADS}_{2}$ score. During the same period of time, the prescription of OACs in patients with a $\mathrm{CHADS}_{2}$ score of $\geq 2$ also increased from $78 \%$ in 2014 to $83 \%$ in 2017. [65] Similar findings were reported from Australia, [43] where OAC prescribing increased from the pre-DOAC era to the post-DOAC era both in low-risk patients (from $35.0 \%$ to 42.9\%, $\mathrm{p}=0.59$ ) and high-risk patients (from 52.2\% to 63.1\%). Despite these significant proportions of overtreatment, it must be noted that the actual numbers of low-risk patients in the Japanese and Australian studies were small. $[43,65]$ Therefore, the primary focus of guideline non-adherence should remain on undertreatment. As shown in Table 1, a significant proportion of patients with AF are still receiving treatment that is not guideline adherent despite gradual improvements over time.

Table 1: Changes in the extent of guideline non-adherence over time in single geographical regions

\begin{tabular}{|c|c|c|c|c|c|}
\hline Study & $\begin{array}{l}\text { Country } \\
\text { (setting) }\end{array}$ & $\begin{array}{c}\text { Guidelines/ } \\
\text { tools }\end{array}$ & $\begin{array}{c}\text { Patient } \\
\text { population }\end{array}$ & $\begin{array}{l}\text { Outcome } \\
\text { measures }\end{array}$ & $\begin{array}{l}\text { Guideline non- } \\
\text { adherence }\end{array}$ \\
\hline $\begin{array}{l}\text { Narita et } \\
\text { al } 2020 \\
{[65]}\end{array}$ & $\begin{array}{l}\text { Japan } \\
\text { (Health } \\
\text { insurance } \\
\text { database) }\end{array}$ & $\mathrm{CHADS}_{2}$ & $\begin{array}{l}\text { Patients with AF } \\
(\mathrm{N}=4375)\end{array}$ & $\begin{array}{l}\text { Prescription of } \\
\text { OACs }\end{array}$ & $\begin{array}{l}\text { Undertreatment in } \\
\text { CHADS }_{2} \text { score of } \geq 2 \text { : } \\
-2014(\mathrm{~N}=708): 22 \% \\
-2015(\mathrm{~N}=702): 20 \% \\
-2016(\mathrm{~N}=688): 21 \% \\
-2017(\mathrm{~N}=758): 17 \% \\
\text { Overtreatment in } \\
\text { CHADS }_{2} \text { score of } 0: \\
-2014(\mathrm{~N}=90): 53 \% \\
-2015(\mathrm{~N}=76): 69 \% \\
-2016(\mathrm{~N}=96): 59 \% \\
-2017(\mathrm{~N}=85): 66 \%\end{array}$ \\
\hline
\end{tabular}




\begin{tabular}{|c|c|c|c|c|c|}
\hline $\begin{array}{l}\text { Cowan et } \\
\text { al 2018 } \\
\text { (The } \\
\text { GRASP- } \\
\text { AF } \\
\text { registry) } \\
\text { [63] }\end{array}$ & $\begin{array}{l}\text { England } \\
\text { (Hospital) }\end{array}$ & $\begin{array}{l}\mathrm{CHA}_{2} \mathrm{DS}_{2-} \\
\mathrm{VASc}\end{array}$ & $\begin{array}{l}\text { Patients with AF } \\
(\mathrm{N}=375310)\end{array}$ & $\begin{array}{l}\text { OAC and AP } \\
\text { use in patients } \\
\text { with } \mathrm{CHA}_{2} \mathrm{DS}_{2} \text { - } \\
\text { VASc } \geq 2 \\
\text { between } 2006 \\
\text { and } 2016\end{array}$ & $\begin{array}{l}\text { Undertreatment with } \\
\text { non-prescription of } \\
\text { OACs: } 52.0 \%(2006) \text { vs } \\
21.4 \%(2016) \\
\text { Undertreatment with } \\
\text { AP: } 42.9 \%(2006) \text { vs } \\
16.1 \%(2016)\end{array}$ \\
\hline $\begin{array}{l}\text { Admassie } \\
\text { et al } 2017 \\
\text { [43] }\end{array}$ & $\begin{array}{l}\text { Australia } \\
\text { (Hospital) }\end{array}$ & $\begin{array}{l}\text { ESC } 2012 \\
\text { and AHA } \\
2014 / \\
\mathrm{CHA}_{2} \mathrm{DS}_{2-} \\
\text { VASc and } \\
\text { HAS- } \\
\text { BLED }\end{array}$ & $\begin{array}{l}\text { Patients with } \\
\text { NVAF (N=2118) }\end{array}$ & $\begin{array}{l}\text { OAC prescribing } \\
\text { in Pre- (Jan } 2011 \\
\text { to Jul 2013) and } \\
\text { post-DOAC era } \\
\text { (Aug } 2013 \text { to Jul } \\
2015 \text { ) }\end{array}$ & $\begin{array}{l}\text { Undertreatment in high- } \\
\text { risk patients: } 44.8 \% \\
\text { (pre-DOAC) vs } 36.9 \% \\
\text { (post-DOAC), p = } \\
0.001 . \\
\text { Overtreatment in low- } \\
\text { risk patients: } 35.0 \% \\
\text { (pre-DOAC) vs } 42.9 \% \\
\text { (post-DOAC), p = } 0.59 .\end{array}$ \\
\hline $\begin{array}{l}\text { Apenteng } \\
\text { et al } 2017 \\
\text { [64] }\end{array}$ & $\begin{array}{l}\text { UK (Primary } \\
\text { care, internal } \\
\text { medicine, } \\
\text { cardiology, } \\
\text { geriatrics, } \\
\text { neurology) }\end{array}$ & $\begin{array}{l}\text { NICE 2014/ } \\
\mathrm{CHA}_{2} \mathrm{DS}_{2^{-}} \\
\text {VASc and } \\
\text { HAS- } \\
\text { BLED }\end{array}$ & $\begin{array}{l}\text { Newly diagnosed } \\
\text { NVAF patients }\end{array}$ & $\begin{array}{l}\text { OAC prescribing } \\
\text { in high-risk } \\
\text { patients } \\
\left(\mathrm{CHA}_{2} \mathrm{DS}_{2^{-}}\right. \\
\text {VASc } \geq 2) \text { and } \\
\text { prescribing no } \\
\text { antithrombotic } \\
\text { medication in } \\
\text { low-risk patients } \\
\left(\mathrm{CHA}_{2} \mathrm{DS}_{2^{-}}\right. \\
\text {VASc=0) } \\
\text { between } \mathrm{Sep} \\
2011 \text { to Apr } \\
2013 \text { and Jul } \\
2015 \text { to Jun } \\
2016 \\
\end{array}$ & $\begin{array}{l}\text { Undertreatment with } \\
\text { non-prescription of } \\
\text { OACs in high-risk: } \\
\text { - Sep } 2011 \text { to Apr 2013: } \\
43.3 \% \\
\text { - Jul } 2015 \text { to Jun 2016: } \\
24.4 \% \\
\text { Overtreatment with } \\
\text { antithrombotic in low- } \\
\text { risk: } \\
\text { - Sep } 2011 \text { to Apr 2013: } \\
78.6 \% \\
\text { - Jul } 2015 \text { to Jun 2016: } \\
64.7 \%\end{array}$ \\
\hline $\begin{array}{l}\text { Lacoin et } \\
\text { al } 2017 \\
{[33]}\end{array}$ & $\begin{array}{l}\text { UK (General } \\
\text { practices) }\end{array}$ & $\begin{array}{l}\text { ESC 2012 } \\
\text { and NICE } \\
2014 / \\
\mathrm{CHA}_{2} \mathrm{DS}_{2-} \\
\mathrm{VASc}\end{array}$ & $\begin{array}{l}\text { Patients with } \\
\mathrm{NVAF} \text { eligible } \\
\text { for } \\
\text { anticoagulation } \\
\left(\mathrm{CHA}_{2} \mathrm{DS}_{2-}\right. \\
\mathrm{VASc} \geq 2) \\
(\mathrm{N}=294786)\end{array}$ & $\begin{array}{l}\text { Patients who } \\
\text { received OACs }\end{array}$ & $\begin{array}{l}\text { Undertreatment: } \\
\text { April } 2012(49.8 \%), \\
\text { April } 2013(46.8 \%), \\
\text { April } 2014(42.5 \%) \text {, } \\
\text { April } 2015(37.1 \%) \text {, and } \\
\text { January } 2016(33.1 \%)\end{array}$ \\
\hline $\begin{array}{l}\text { Marzec et } \\
\text { al } 2017 \\
{[37]}\end{array}$ & $\begin{array}{l}\text { U.S. } \\
\text { (Ambulatory) }\end{array}$ & $\begin{array}{l}\mathrm{CHA}_{2} \mathrm{DS}_{2-} \\
\text { VASc }\end{array}$ & $\begin{array}{l}\text { Patients with } \\
\mathrm{NVAF} \text { and high } \\
\text { risk for stroke } \\
\text { (i.e. } \mathrm{CHA}_{2} \mathrm{DS}_{2-} \\
\text { VASc score } \geq 2 \text { ) } \\
\text { without a } \\
\text { documented } \\
\text { contraindication } \\
\text { for OAC } \\
(\mathrm{N}=655000) \\
\end{array}$ & OAC use & $\begin{array}{l}\text { Undertreatment: } \\
\text { 2008: } 47.6 \% \\
\text { 2014: } 39.3 \% \text { (p for trend } \\
<0.01 \text { ) }\end{array}$ \\
\hline $\begin{array}{l}\text { Mochalina } \\
\text { et al } 2016 \\
\text { [53] }\end{array}$ & $\begin{array}{l}\text { Sweden } \\
\text { (Primary and } \\
\text { secondary } \\
\text { care) }\end{array}$ & $\begin{array}{l}\text { ESC 2012/ } \\
\mathrm{CHA}_{2} \mathrm{DS}_{2-} \\
\text { VASc and } \\
\text { HAS- } \\
\text { BLED }\end{array}$ & $\begin{array}{l}\text { Patients with first } \\
\text { episode AF } \\
(\mathrm{N}=13837)\end{array}$ & $\begin{array}{l}\text { Guideline } \\
\text { adherence; } \\
\text { potential } \\
\text { undertreatment; } \\
\text { and } \\
\text { overtreatment, } \\
\text { respectively }\end{array}$ & $\begin{array}{l}\text { Guideline non-adherent } \\
\text { treatment: } \\
\text { 2011: } 52.4 \% \\
\text { 2012: } 47.0 \% \\
\text { 2013: } 40.2 \% \\
\text { 2014: } 33.9 \% \\
\text { Undertreatment: } \\
\text { 2011: } 51.9 \% \\
\text { 2012: } 46.3 \% \\
\text { 2013: } 39.6 \% \\
\text { 2014: } 33.3 \%\end{array}$ \\
\hline
\end{tabular}




\begin{tabular}{|l|l|l|l|l|}
\hline & & & & Overtreatment: \\
& & & $2011: 0.6 \%$ \\
& & & $2012: 0.7 \%$ \\
& & & $2013: 0.6 \%$ \\
& & & $2014: 0.6 \%$ \\
\hline
\end{tabular}

AF: atrial fibrillation; AHA: American Heart Association; AP: antiplatelet agents; $\mathrm{CHA}_{2} \mathrm{DS}_{2}$-VASc: congestive heart failure, hypertension, age $\geq 75$ (doubled), diabetes, stroke (doubled)-vascular disease, age 65-74, and sex category (female); $\mathrm{CHADS}_{2}$ : congestive heart failure, hypertension, age $\geq 75$ years, diabetes, a history of stroke or transient ischemic attack (doubled); ESC: European Society of Cardiology; GRASP: Guidance on Risk Assessment for Stroke Prevention in AF; HAS-BLED: Hypertension, Abnormal renal/liver function, Stroke, Bleeding history or predisposition, Labile international normalized ratio, Elderly (>65 years), Drugs/alcohol concomitantly; NICE: National Institute for Health and Care Excellence; NVAF: nonvalvular atrial fibrillation; OAC: oral anticoagulant; U.K.: United Kingdom; U.S.: United States

Studies that included patients from multiple geographical regions demonstrated variations in the extent of guideline non-adherence between regions. Studies from the Global Registry on Long-Term Oral Antithrombotic Treatment in Patients with Atrial Fibrillation (GLORIA-AF) registry show that undertreatment in terms of not prescribing OACs for patients with a $\mathrm{CHA}_{2} \mathrm{DS}_{2}$-VASc score of $\geq 2$ is lowest in Europe and highest in Asia. [24, 25] Mazurek et al also reported that undertreatment in Africa/Middle East is lowest second only to Europe. [24] However, with only 32 (of 536) participants from South Africa, it is difficult to generalize this to the entire African population (Table 2).

Table 2: The extent of guideline non-adherence in studies conducted in multiple geographical regions

\begin{tabular}{|c|c|c|c|c|c|}
\hline $\begin{array}{l}\text { Study } \\
\text { (prospective }\end{array}$ & $\begin{array}{l}\text { Country } \\
\text { (Setting) }\end{array}$ & $\begin{array}{l}\text { Guidelines/ } \\
\text { tools }\end{array}$ & $\begin{array}{l}\text { Patient } \\
\text { population }\end{array}$ & $\begin{array}{l}\text { Outcome } \\
\text { measures }\end{array}$ & Results \\
\hline $\begin{array}{l}\text { Miyazawa et } \\
\text { al (Fushimi } \\
\text { and } \\
\text { Darlington } \\
\text { AF } \\
\text { registries) } \\
2019[26]\end{array}$ & $\begin{array}{l}\text { Japan and } \\
\text { U.K. } \\
\text { (Community- } \\
\text { based in- } \\
\text { patient and } \\
\text { outpatient } \\
\text { services; and } \\
\text { general } \\
\text { practices) }\end{array}$ & $\begin{array}{l}\text { Fushimi: JCS } \\
\text { 2013/ CHADS } \\
\text { Darlington: } \\
\text { NICE 2014/ } \\
\mathrm{CHA}_{2} \mathrm{DS}_{2-} \\
\text { VASc }\end{array}$ & $\begin{array}{l}\text { Patients with } \\
\text { AF or atrial } \\
\text { flutter } \\
(\mathrm{N}=6244)\end{array}$ & $\begin{array}{l}\text { Guideline non- } \\
\text { adherence }\end{array}$ & $\begin{array}{l}\text { Non-adherent } \\
\text { (p<0.001): } \\
\text {-Fushimi: } 41.4 \% \\
\text {-Darlington: } 49.2 \% \\
\text { Undertreatment } \\
\text { (p=0.002): } \\
\text {-Fushimi: } 40.0 \% \\
\text {-Darlington: } 36.1 \% \\
\text { Overtreatment } \\
\text { (p<0.001): } \\
\text {-Fushimi: } 1.4 \% \\
\text {-Darlington: } 13.1 \%\end{array}$ \\
\hline $\begin{array}{l}\text { Mazurek et } \\
\text { al (The } \\
\text { GLORIA- } \\
\text { AF Registry, } \\
\text { Phase II) } \\
2017 \text { [24] }\end{array}$ & $\begin{array}{l}\text { Asia, Europe, } \\
\text { North } \\
\text { America, } \\
\text { Latin } \\
\text { America, } \\
\text { Africa/Middle } \\
\text { East } \\
\text { (inpatient and } \\
\text { outpatient) }\end{array}$ & $\begin{array}{l}\mathrm{CHA}_{2} \mathrm{DS}_{2^{-}} \\
\text {VASc }\end{array}$ & $\begin{array}{l}\text { Adult NVAF } \\
\text { patients with } \\
\geq 1 \text { risk factor } \\
\text { for stroke as } \\
\text { per } \\
\mathrm{CHA}_{2} \mathrm{DS}_{2-} \\
\text { VASc } \\
(\mathrm{N}=12999)\end{array}$ & $\begin{array}{l}\text { OAC non- } \\
\text { prescription in } \\
\text { patients with } \\
\mathrm{CHA}_{2} \mathrm{DS}_{2-} \\
\text { VASc score of } \\
\geq 2\end{array}$ & $\begin{array}{l}\text { Entire population } \\
(\mathrm{N}=12999): 17.8 \% \\
\text { Asia }(\mathrm{N}=2429): \\
42.4 \% \\
\text { Europe }(\mathrm{N}=6310) \text { : } \\
8.9 \% \\
\text { North America } \\
(\mathrm{N}=2933): 19.2 \% \\
\text { Latin America } \\
(\mathrm{N}=791): 13.3 \% \\
\text { Africa/Middle East* } \\
(\mathrm{N}=536): 10.8 \%\end{array}$ \\
\hline $\begin{array}{l}\text { Huisman et } \\
\text { al (The } \\
\text { GLORIA- }\end{array}$ & $\begin{array}{l}\text { Asia, Europe, } \\
\text { North } \\
\text { America, }\end{array}$ & $\begin{array}{l}\mathrm{CHA}_{2} \mathrm{DS}_{2-} \\
\mathrm{VASc}\end{array}$ & $\begin{array}{l}\text { Adult NVAF } \\
\text { patients with } \\
\geq 1 \text { risk factor }\end{array}$ & $\begin{array}{l}\text { OAC non- } \\
\text { prescription in } \\
\text { patients with }\end{array}$ & $\begin{array}{l}\text { Entire population } \\
(\mathrm{N}=10675): 16.7 \%\end{array}$ \\
\hline
\end{tabular}




\begin{tabular}{|c|c|c|c|c|}
\hline $\begin{array}{l}\text { AF Registry, } \\
\text { Phase II) } \\
2015 \text { [25] }\end{array}$ & $\begin{array}{l}\text { Latin } \\
\text { America, } \\
\text { Africa/Middle } \\
\text { East } \\
\text { (inpatient and } \\
\text { outpatient) }\end{array}$ & $\begin{array}{l}\text { for stroke as } \\
\text { per } \\
\mathrm{CHA}_{2} \mathrm{DS}_{2-} \\
\text { VASc } \\
(\mathrm{N}=10675)\end{array}$ & $\begin{array}{l}\mathrm{CHA}_{2} \mathrm{DS}_{2-} \\
\text { VASc score of } \\
\geq 2\end{array}$ & $\begin{array}{l}\text { Asia }(\mathrm{N}=1957) \text { : } \\
39.3 \% \\
\text { Europe }(\mathrm{N}=4703) \text { : } \\
8.9 \% \\
\text { North America } \\
(\mathrm{N}=3415): 19.3 \% \\
\text { Latin America } \\
(\mathrm{N}=476): 13.9 \% \\
\text { Africa/ Middle East } \\
(\mathrm{N}=124): \text { not stated }\end{array}$ \\
\hline
\end{tabular}

*Africa/Middle East: only 32 patients from South Africa; AF: atrial fibrillation; $\mathrm{CHA}_{2} \mathrm{DS}_{2}-\mathrm{VASc}$ : congestive heart failure, hypertension, age $\geq 75$ (doubled), diabetes, stroke (doubled)-vascular disease, age 65-74, and sex category (female); $\mathrm{CHADS}_{2}$ : congestive heart failure, hypertension, age $\geq 75$ years, diabetes, a history of stroke or transient ischemic attack (doubled); GLORIA-AF: Global Registry on Long-Term Oral Antithrombotic Treatment in Patients with Atrial Fibrillation; JCS: Japanese Circulation Society; NICE: National Institute for Health and Care Excellence; NVAF: non-valvular atrial fibrillation; OAC: oral anticoagulant

Although there are several studies which included participants from multiple countries or regions, few of them compared overall results across the different regions. Table 3 focuses on studies that were published between 2016 and 2020 and that used the $\mathrm{CHA}_{2} \mathrm{DS}_{2}$-VASc score as a means of stratifying patients' risk of stroke. Significant differences in guideline non-adherence exist across and within different geographic regions (Table 3).

Table 3: The extent of guideline non-adherence in different geographical regions and healthcare settings in the past five years

\begin{tabular}{|c|c|c|c|c|c|}
\hline & $\begin{array}{l}\text { Setting } \\
\text { (Country) }\end{array}$ & Patient population & $\begin{array}{l}\text { Guideline- } \\
\text { adherent } \\
\text { treatment }\end{array}$ & Undertreatment & Overtreatment \\
\hline \multicolumn{6}{|l|}{ Middle East } \\
\hline $\begin{array}{l}\text { Miyazawa et al } \\
2019^{\&} 2[66] \\
\end{array}$ & $\begin{array}{l}\text { Hospital (Gulf } \\
\text { countries) }\end{array}$ & $\begin{array}{l}\text { Patients with AF } \\
(\mathrm{N}=1860)\end{array}$ & $49 \%$ & $25.6 \%$ & $25.4 \%$ \\
\hline $\begin{array}{l}\text { Balaghi-Inalou } \\
\text { et al } 2017^{\#}[30]\end{array}$ & Hospital (Iran) & $\begin{array}{l}\text { Patients with NVAF } \\
(\mathrm{N}=120)\end{array}$ & $97.5 \%$ & - & - \\
\hline $\begin{array}{l}\text { Kezerle et al } \\
2020^{\lambda}[62]\end{array}$ & $\begin{array}{l}\text { Community } \\
\text { and hospital } \\
\text { (Israel) }\end{array}$ & $\begin{array}{l}\text { Patients with NVAF } \\
(\mathrm{N}=58385)\end{array}$ & $37.1 \%^{2}$ & $62.9 \%^{2}$ & $11.4 \%^{0}$ \\
\hline \multicolumn{6}{|l|}{ East Asia } \\
\hline $\begin{array}{l}\text { Krittayaphong } \\
\text { et al } 2018^{\&} \\
{[50]}\end{array}$ & $\begin{array}{l}\text { Hospital } \\
\text { (Thailand) }\end{array}$ & $\begin{array}{l}\text { Patients with NVAF } \\
(\mathrm{N}=3218)\end{array}$ & $81.6 \%{ }^{2}$ & $18.4 \%^{2}$ & $58.4 \%^{0}$ \\
\hline $\begin{array}{l}\text { Kim et al } \\
2017^{\&}[59]\end{array}$ & $\begin{array}{l}\text { Hospital } \\
\text { (South Korea) }\end{array}$ & $\begin{array}{l}\text { Patients with NVAF } \\
(\mathrm{N}=6275)\end{array}$ & $82.7 \%^{2}$ & $17.3 \%^{2}$ & $53.4 \%^{0}$ \\
\hline $\begin{array}{l}\text { Song et al } \\
2019^{\lambda}[67]\end{array}$ & $\begin{array}{l}\text { Inpatient and } \\
\text { outpatient } \\
\text { (South Korea) }\end{array}$ & $\begin{array}{l}\text { Patients with NVAF } \\
(\mathrm{N}=9226)\end{array}$ & $54.2 \%{ }^{2}$ & $45.8 \%{ }^{2}$ & - \\
\hline $\begin{array}{l}\text { Lee et al } 2017^{\lambda} \\
{[34]}\end{array}$ & $\begin{array}{l}\text { Claims } \\
\text { database } \\
\text { (South Korea) }\end{array}$ & $\begin{array}{l}\text { Patients with NVAF } \\
(\mathrm{N}=276246)\end{array}$ & $50.6 \%{ }^{2}$ & $49.4 \%{ }^{2}$ & - \\
\hline \multicolumn{6}{|l|}{ Australasia } \\
\hline $\begin{array}{l}\text { Wertheimer et } \\
\text { al } 2020^{\lambda}[57]\end{array}$ & $\begin{array}{l}\text { Hospital } \\
\text { (Australia) }\end{array}$ & $\begin{array}{l}\text { Patients with AF } \\
(\mathrm{N}=200)\end{array}$ & $72.8 \%{ }^{2}$ & $27.2 \%^{2}$ & $80 \%^{0}$ \\
\hline $\begin{array}{l}\text { Admassie et al } \\
2017^{\lambda}[43]\end{array}$ & $\begin{array}{l}\text { Hospital } \\
\text { (Australia) }\end{array}$ & $\begin{array}{l}\text { Patients with NVAF } \\
(\mathrm{N}=2118)\end{array}$ & $63.1 \%{ }^{2}$ & $36.9 \%{ }^{2}$ & $42.9 \%{ }^{0}$ \\
\hline $\begin{array}{l}\text { Bista et al } \\
2017^{\lambda}[68]\end{array}$ & $\begin{array}{l}\text { Hospital } \\
\text { (Australia) }\end{array}$ & $\begin{array}{l}\text { Patients with AF } \\
(\mathrm{N}=1469)\end{array}$ & $57 \%^{1 \mathrm{c}}$ & $43 \%^{1 \mathrm{c}}$ & - \\
\hline
\end{tabular}




\begin{tabular}{|c|c|c|c|c|c|}
\hline $\begin{array}{l}\text { Aziz et al } \\
2019^{\lambda}[69]\end{array}$ & $\begin{array}{l}\text { Remote } \\
\text { hospital } \\
\text { (Australia) }\end{array}$ & $\begin{array}{l}\text { Patients with } \mathrm{AF} \text { or } \\
\text { atrial flutter }(\mathrm{N}=59)\end{array}$ & $83 \%{ }^{2}$ & $17 \%^{2}$ & - \\
\hline $\begin{array}{l}\text { Frain et al } \\
2018^{\lambda}[70]\end{array}$ & $\begin{array}{l}\text { Aged care } \\
\text { (Australia) }\end{array}$ & $\begin{array}{l}\text { Patients with AF } \\
\text { residing in aged care } \\
\text { facilities }(N=1952)\end{array}$ & $34.8 \%^{\mathrm{la}}$ & $65.2 \%^{1 \mathrm{~b}}$ & - \\
\hline $\begin{array}{l}\text { Olivia et al } \\
2020^{\lambda}[71]\end{array}$ & $\begin{array}{l}\text { Nurse-led } \\
\text { clinic } \\
\text { (Australia) }\end{array}$ & $\begin{array}{l}\text { Patients with AF } \\
(\mathrm{N}=136)\end{array}$ & $91.3 \%{ }^{2}$ & $8.7 \%^{2}$ & - \\
\hline $\begin{array}{l}\text { Tomlin et al } \\
2017^{\lambda}[18]\end{array}$ & $\begin{array}{l}\text { General } \\
\text { practices (New } \\
\text { Zealand) } \\
\end{array}$ & $\begin{array}{l}\text { Patients with } A F \\
(\mathrm{~N}=12712)\end{array}$ & $60.5 \%{ }^{2}$ & $39.5 \%{ }^{2}$ & $15.6 \%^{0}$ \\
\hline \multicolumn{6}{|l|}{ Europe } \\
\hline $\begin{array}{l}\text { Kartas et al } \\
2019^{\#}[49]\end{array}$ & $\begin{array}{l}\text { Hospital } \\
\text { (Greece) }\end{array}$ & $\begin{array}{l}\text { Patients with NVAF } \\
(\mathrm{N}=768)\end{array}$ & $\begin{array}{l}83.1 \% \\
85.4 \%{ }^{2}\end{array}$ & $14.6 \%{ }^{2}$ & $67.6 \%{ }^{0}$ \\
\hline $\begin{array}{lll}\text { Jortveit et al } \\
2019^{\&}[72] & \end{array}$ & $\begin{array}{l}\text { Hospital } \\
\text { (Norway) }\end{array}$ & $\begin{array}{l}\text { Patients with acute MI } \\
\text { and } A F(N=7583)\end{array}$ & $56.3 \%{ }^{2}$ & $43.7 \%^{2}$ & - \\
\hline $\begin{array}{l}\text { Cowan et al } \\
2018^{\lambda}[63]\end{array}$ & $\begin{array}{l}\text { Hospital } \\
\text { (England) }\end{array}$ & $\begin{array}{l}\text { Patients with AF } \\
(\mathrm{N}=375310)\end{array}$ & $78.6 \%{ }^{2}$ & $21.4 \%^{2}$ & - \\
\hline $\begin{array}{l}\text { Gundlund et al } \\
2018^{\lambda} \text { [73] }\end{array}$ & $\begin{array}{l}\text { Hospital } \\
\text { (Denmark) }\end{array}$ & $\begin{array}{l}\text { Patients with NVAF } \\
\text { aged } \geq 30 \text { years, with } \\
\text { moderate to high } \\
\text { stroke risk }(N=30626)\end{array}$ & $\begin{array}{l}\text { Pre-stroke } \\
\text { population: } \\
36.5 \%^{2} \\
\text { Post-stroke } \\
\text { population: } \\
54.2 \% \%^{2} \\
\end{array}$ & $\begin{array}{l}\text { Pre-stroke } \\
\text { population: } \\
63.5 \%{ }^{2} \\
\text { Post-stroke } \\
\text { population: } \\
45.8 \%{ }^{2} \\
\end{array}$ & - \\
\hline $\begin{array}{l}\text { Averlant et al } \\
2017^{\lambda}[41]\end{array}$ & $\begin{array}{l}\text { Hospital } \\
\text { (France) }\end{array}$ & $\begin{array}{l}\text { Patients with NVAF } \\
\text { aged } \geq 75(\mathrm{~N}=2034)\end{array}$ & $41.5 \%$ & $58.5 \%$ & - \\
\hline $\begin{array}{l}\text { Başaran et al } \\
2017^{\lambda}[20]\end{array}$ & $\begin{array}{l}\text { Hospital } \\
\text { (Turkey) }\end{array}$ & $\begin{array}{l}\text { Patients with NVAF } \\
(\mathrm{N}=6273)\end{array}$ & $71.7 \%$ & $23.9 \%$ & $4.4 \%$ \\
\hline $\begin{array}{l}\text { Proietti et al } \\
2016^{\&}[74]\end{array}$ & $\begin{array}{l}\text { Hospital (Italy } \\
\text { and Spain) }\end{array}$ & $\begin{array}{l}\text { Patients with AF aged } \\
\geq 65 \text { years }(\mathrm{N}=558)\end{array}$ & $40.9 \%$ & $52.3 \%$ & $6.8 \%$ \\
\hline $\begin{array}{l}\text { Lenarczyk et al } \\
2016^{\&}[51]\end{array}$ & $\begin{array}{l}\text { Hospital } \\
\text { (Poland) }\end{array}$ & $\begin{array}{l}\text { Patients with AF } \\
(\mathrm{N}=419)\end{array}$ & $61.1 \%^{2}$ & $38.9 \%^{2}$ & $66.7 \%^{0}$ \\
\hline $\begin{array}{lrr}\text { Seelig et } & \text { al } \\
2020^{\&} & {[56]}\end{array}$ & $\begin{array}{l}\text { Hospital, } \\
\text { primary care, } \\
\text { anticoagulation } \\
\text { clinics } \\
\text { (Netherlands) }\end{array}$ & $\begin{array}{l}\text { Patients with NVAF } \\
(\mathrm{N}=1189)\end{array}$ & $\begin{array}{l}82.6 \%^{1 \mathrm{a} a} \text { to } \\
92.5 \%^{2}\end{array}$ & $\begin{array}{l}7.5^{2} \text { to } \\
17.8 \% \%^{1 a}\end{array}$ & $71.1 \%^{0}$ \\
\hline $\begin{array}{lrr}\text { Cools et } & \text { al } \\
2018^{\&}[46] & \end{array}$ & $\begin{array}{l}\text { Hospital } \\
\text { cardiology } \\
\text { sites, private } \\
\text { practice } \\
\text { cardiology site, } \\
\text { general } \\
\text { physician sites } \\
\text { (Belgium) }\end{array}$ & $\begin{array}{l}\text { Newly diagnosed } \\
\text { NVAF patients with } \\
\text { at least one stroke risk } \\
\text { factor }(\mathrm{N}=1713)\end{array}$ & $84.3 \%{ }^{2}$ & $15.7 \%^{2}$ & $71.4 \%^{0}$ \\
\hline $\begin{array}{l}\text { Apenteng et al } \\
2017^{\&} \text { [64] }\end{array}$ & $\begin{array}{l}\text { Primary care, } \\
\text { internal } \\
\text { medicine, } \\
\text { cardiology, } \\
\text { geriatrics, } \\
\text { neurology } \\
\text { (U.K.) }\end{array}$ & $\begin{array}{l}\text { Newly diagnosed } \\
\text { NVAF patients } \\
(\mathrm{N}=3482)\end{array}$ & $75.6 \%{ }^{2}$ & $24.4 \%^{2}$ & $64.7 \%$ \\
\hline $\begin{array}{l}\text { Potpara et al } \\
2016^{\&}[54]\end{array}$ & $\begin{array}{l}\text { Multiple } \\
\text { settings } \\
\text { (Balkan } \\
\text { countries) }\end{array}$ & $\begin{array}{l}\text { Patients with AF } \\
(\mathrm{N}=2663)\end{array}$ & $74.4 \%^{2}$ & $25.6 \%{ }^{2}$ & $50.38 \% 0$ \\
\hline $\begin{array}{l}\text { Mazurek et al } \\
2017^{\&} 2[75]\end{array}$ & $\begin{array}{l}\text { Primary health } \\
\text { care (U.K.) }\end{array}$ & $\begin{array}{l}\text { Patients with AF or } \\
\text { atrial flutter }(\mathrm{N}=2259)\end{array}$ & $50.8 \%$ & $36.1 \%$ & $13.1 \%$ \\
\hline
\end{tabular}




\begin{tabular}{|c|c|c|c|c|c|}
\hline $\begin{array}{l}\text { Lacoin et al } \\
2017^{\#}[33]\end{array}$ & $\begin{array}{l}\text { General } \\
\text { practices } \\
\text { (U.K.) }\end{array}$ & $\begin{array}{l}\text { Patients with NVAF } \\
\text { eligible for } \\
\text { anticoagulation } \\
\left(\mathrm{CHA}_{2} \mathrm{DS}_{2}-\mathrm{VASc} \geq 2\right) \\
(\mathrm{N}=294786)\end{array}$ & $66.9 \%^{2}$ & $33.1 \%^{2}$ & - \\
\hline $\begin{array}{l}\text { Mochalina et al } \\
2016^{\lambda}[53]\end{array}$ & $\begin{array}{l}\text { Primary and } \\
\text { secondary care } \\
\text { (Sweden) }\end{array}$ & $\begin{array}{l}\text { Patients with first } \\
\text { NVAF or atrial flutter } \\
(\mathrm{N}=13837)\end{array}$ & $62.8 \%^{2}$ & $37.2 \%^{2}$ & - \\
\hline $\begin{array}{l}\text { Boriani et al } \\
2018^{\&}[45]\end{array}$ & $\begin{array}{l}\text { Cardiology } \\
\text { practices } \\
\text { (Multicountry) }\end{array}$ & $\begin{array}{l}\text { Patients with AF } \\
(\mathrm{N}=11096)\end{array}$ & $\begin{array}{l}78.3- \\
88.8 \%^{2}\end{array}$ & $11.2-21.7 \%^{2}$ & $73.5 \%^{0}$ \\
\hline $\begin{array}{l}\text { Navarro-Juan } \\
\text { et al 2016 } 6^{\#}[76]\end{array}$ & $\begin{array}{l}\text { Emergency } \\
\text { department } \\
\text { (Spain) }\end{array}$ & $\begin{array}{l}\text { Patients with AF } \\
(\mathrm{N}=144)\end{array}$ & $33.1 \%^{2}$ & $66.9 \%^{2}$ & $16.7 \%^{0}$ \\
\hline $\begin{array}{l}\text { Hohnloser et al } \\
2019^{\lambda}[22]\end{array}$ & $\begin{array}{l}\text { Claims } \\
\text { database } \\
\text { (Germany) }\end{array}$ & $\begin{array}{l}\text { Patients with NVAF } \\
(\mathrm{N}=601261)\end{array}$ & $54.2 \%^{2}$ & $45.8 \%^{2}$ & $29.6 \%^{0}$ \\
\hline \multicolumn{6}{|l|}{$\begin{array}{l}\text { North } \\
\text { America }\end{array}$} \\
\hline $\begin{array}{l}\text { Piccini et } \\
2019^{\&}[38]\end{array}$ & Hospital (U.S.) & $\begin{array}{l}\text { Patients with NVAF, } \\
\mathrm{CHA}_{2} \mathrm{DS}_{2} \text {-VASc } \\
\text { score } \geq 2 \text {, and without } \\
\text { a documented } \\
\text { contraindication for } \\
\mathrm{OAC}(\mathrm{N}=27270)\end{array}$ & $80.3 \%^{2}$ & $19.7 \%^{2}$ & - \\
\hline $\begin{array}{l}\text { Dupree et al } \\
2018^{\lambda}[47]\end{array}$ & Hospital (U.S.) & $\begin{array}{l}\text { Patients with NVAF } \\
\text { without a documented } \\
\text { contraindication for } \\
\mathrm{OAC}(\mathrm{N}=246)\end{array}$ & $83.0 \%^{2}$ & $17.0 \%{ }^{2}$ & $83.3 \%^{0}$ \\
\hline $\begin{array}{l}\text { McIntyre et al } \\
2018^{\&}[77]\end{array}$ & $\begin{array}{l}\text { Hospitals as } \\
\text { well as the } \\
\text { offices of } \\
\text { specialists and } \\
\text { primary care } \\
\text { providers (U.S. } \\
\text { and Canada) }\end{array}$ & $\begin{array}{l}\text { Newly diagnosed } \\
\text { patients with } \mathrm{AF} \text { with } \\
\text { a } \mathrm{CHA}_{2} \mathrm{DS}_{2}-\mathrm{VASc} \geq 1 \\
\text { in males and } \geq 2 \text { in } \\
\text { females }(\mathrm{N}=3320)\end{array}$ & $83 \% \%^{2}$ & $17 \%^{2}$ & - \\
\hline $\begin{array}{lrr}\text { Lubitz et } & \text { al } \\
2018^{\&}[36] & \end{array}$ & $\begin{array}{l}\text { Ambulatory } \\
\text { (U.S.) }\end{array}$ & $\begin{array}{l}\text { Patients with NVAF } \\
\text { and high risk for } \\
\text { stroke (i.e. } \mathrm{CHA}_{2} \mathrm{DS}_{2-} \\
\text { VASc score } \geq 2) \\
(\mathrm{N}=674841)\end{array}$ & $57 \%^{2}$ & $43 \%^{2}$ & - \\
\hline $\begin{array}{l}\text { Marzec et al } \\
2017^{\&}[37]\end{array}$ & $\begin{array}{l}\text { Ambulatory } \\
\text { (U.S.) }\end{array}$ & $\begin{array}{l}\text { Patients with NVAF } \\
\text { and high risk for } \\
\text { stroke }\left(\mathrm{CHA}_{2} \mathrm{DS}_{2-}\right. \\
\text { VASc score } \geq 2) \\
\text { without a documented } \\
\text { contraindication for } \\
\text { OAC }(\mathrm{N}=655000)\end{array}$ & $60.7 \%^{2}$ & $39.3 \%{ }^{2}$ & - \\
\hline $\begin{array}{l}\text { Thompson et al } \\
2017^{\&}[39]\end{array}$ & $\begin{array}{l}\text { Ambulatory } \\
\text { (U.S.) }\end{array}$ & $\begin{array}{l}\text { Patients with first } \\
\text { NVAF at high stroke } \\
\text { risk }\left(\mathrm{CHA}_{2} \mathrm{DS}_{2} \text {-VASc }\right. \\
\text { score } \geq 2)(\mathrm{N}=691906)\end{array}$ & $59.1 \%^{2}$ & $40.9 \%^{2}$ & - \\
\hline $\begin{array}{l}\text { Barnett et al } \\
2017^{\lambda}[44]\end{array}$ & $\begin{array}{l}\text { Community- } \\
\text { based } \\
\text { outpatient } \\
\text { practices } \\
\text { (U.S.) }\end{array}$ & $\begin{array}{l}\text { Patients with AF } \\
(\mathrm{N}=9570)\end{array}$ & $78.9 \%^{2}$ & $21.1 \%^{2}$ & $60 \%^{0}$ \\
\hline Africa & & & & & \\
\hline
\end{tabular}




\begin{tabular}{|l|l|l|l|l|l|}
\hline $\begin{array}{l}\text { Gebreyohannes } \\
\text { et al 2018 }[78]\end{array}$ & $\begin{array}{l}\text { Hospital } \\
\text { (Ethiopia) }\end{array}$ & $\begin{array}{l}\text { Patients with AF } \\
(\mathrm{N}=159)\end{array}$ & $35.2 \%$ & $64.8 \%$ & - \\
\hline Latin America & & & & & \\
\hline $\begin{array}{l}\text { Jerjes-Sanchez } \\
\text { et al 2019\& }[48]\end{array}$ & $\begin{array}{l}\text { Multiple } \\
\text { practices } \\
\text { (Argentina, } \\
\text { Chile, Brazil, } \\
\text { Mexico) }\end{array}$ & $\begin{array}{l}\text { NVAly patients with at } \\
\text { least one stroke risk } \\
\text { factor (N=4162) }\end{array}$ & $49.9 \%^{2}$ & $50.1 \%^{2}$ & $63.5 \%^{0}$ \\
\hline
\end{tabular}

${ }^{0}$ Patients who are truly low-risk for stroke (a $\mathrm{CHA}_{2} \mathrm{DS}_{2}$-VASc scores of 0 in men and 1 in women); ${ }^{\text {la }}$ Patients with a $\mathrm{CHA}_{2} \mathrm{DS}_{2}$-VASc scores of 1 in men and 2 in women; ${ }^{1 \mathrm{~b}}$ Patients with a $\mathrm{CHA}_{2} \mathrm{DS}_{2}$-VASc scores of $\geq 1$ in men and $\geq 2$ in women; 1c Patients with a $\mathrm{CHA}_{2} \mathrm{DS}_{2}$-VASc scores of $\geq 1$; ${ }^{2}$ Patients at high-risk for stroke; ${ }^{\#}$ Cross-sectional; \&Prospective cohort; ${ }^{\lambda}$ Retrospective observational; $\mathrm{AF}$ : atrial fibrillation; $\mathrm{CHA}_{2} \mathrm{DS}_{2}-\mathrm{VASc}$ : congestive heart failure, hypertension, age $\geq 75$ (doubled), diabetes, stroke (doubled)-vascular disease, age 65-74, and sex category (female); Balkan countries: Albania, Bosnia-Herzegovina, Bulgaria, Croatia, Montenegro, Romania and Serbia; Gulf countries: Bahrain, Kuwait, Qatar, Oman, The United Arab Emirates, and Yemen; MI: myocardial infarction; NVAF: non-valvular atrial fibrillation; U.K.: the United Kingdom; U.S.: the United States

\subsection{Middle East}

Three studies from the Middle East reported guideline non-adherence. [30, 62, 66] One study from Iran reported that only $2.5 \%$ of patients received guideline non-adherent treatment. [30] Compared to the other two studies [62, 66], this study had a lower sample size. In addition, unlike the other two studies, this study also considered the HAS-BLED score in judging the appropriateness of OAC prescription. This means, individuals that would have been considered receiving undertreatment as per their $\mathrm{CHA}_{2} \mathrm{DS}_{2}-\mathrm{VASc}$ score could be classified as receiving guideline-adherent treatment because of their HAS-BLED score. This could have contributed to the very low proportion of patients with guideline non-adherent treatment. On the other hand, a recent hospital-based study conducted in six Gulf countries reported that nearly half of the patients received guideline non-adherent treatment, with patients receiving over- $(25.6 \%)$ and undertreatment $(25.4 \%)$ in similar rates. [66] This study did not consider the HAS-BLED score but omission of OACs were considered appropriate in patients at high risk of stroke when contraindications were reported. A study in Israel also reported undertreatment as high as 62.9\%. [62] This study assessed the prescription of OACs only in the first three months of AF diagnosis. As such, OACs prescribed after the first three months of treatment would not be considered in assessing guideline adherence. In this study, $11.4 \%$ of patients who were truly at low-risk for stroke received prescription of antithrombotic agents. [62] However, the study did not consider the use of antiplatelet agents in low-risk patients as overtreatment. [62]

\subsection{East Asia}

When considering only patients with a $\mathrm{CHA}_{2} \mathrm{DS}_{2}-\mathrm{VASc}$ score of $\geq 2$, undertreatment in East Asia was reported to be as low as $17.3 \%$ or as high as $49.4 \%$. [34, 50, 59, 67] Similar rates of undertreatment was reported from two hospital-based studies in Thailand and South Korea. [50, 59] On the other hand, two other studies from South Korea reported that $45.8 \%$ to $49.4 \%$ of patients received undertreatment. [34, 67] Of note, the reported rates of 
217 undertreatment was much higher in the two retrospective observational studies [34, 67] than in the other two 218 prospective ones [50, 59], indicating that retrospective studies may overestimate guideline non-adherence. When 219 it comes to overtreatment, prescription of antithrombotic agents in patients who were truly at low-risk for stroke ranged from $53.4 \%$ to $58.4 \%$. [50, 59] It is difficult to make comparisons between the different healthcare settings as most of the participants were either from the hospital setting or from a claims database. $[34,50,59,67]$

\subsection{Australasia}

Undertreatment in Australian patients with a $\mathrm{CHA}_{2} \mathrm{DS}_{2}$-VASc scores of $\geq 2$ prescribed OACs after hospital attendance range from $17.0 \%$ to $36.9 \%$. [43, 57, 69] One study reported that around $40 \%$ of patients who are truly low-risk for stroke were overtreated. [43] Another study reported a higher figure of overtreatment (80\%), but it included a small number of low-risk patients $(\mathrm{N}=16)$. [57] Undertreatment in New Zealand general practices among patients with $\mathrm{CHA}_{2} \mathrm{DS}_{2}$-VASc scores of $\geq 2$ was around $40 \%$ with overtreatment in patients with a $\mathrm{CHA}_{2} \mathrm{DS}_{2}$-VASc scores of 0 of $15.6 \%$. [18] The lowest extent of undertreatment was reported from a nurse-led clinic in Australia where $8.7 \%$ of patients with $\mathrm{CHA}_{2} \mathrm{DS}_{2}$-VASc scores of $\geq 2$ did not receive OAC prescription. [71] Of note, findings from all of these retrospective studies suggest that studies with lower sample size my underestimate the proportion of patients receiving undertreatment. $[18,43,57,69]$ With the exception of the nurse-led clinic [71], an increasing trend in the proportion of patients receiving undertreatment was observed with increasing sample size. [18, 43, 57, 69]

\subsection{Europe}

Of all the regions, Europe is the most frequently studied. In all studies that provided data for high-risk patients, undertreatment was considered when OACs were not prescribed in these patients. $[22,33,41,45,46,49,51,53$, $54,56,63,64,72,73,76]$ Similarly, prescription of any antithrombotic agent in patients with a low risk of stroke was considered as overtreatment. [22, 45, 46, 51, 54, 56, 76] received guideline non-adherent treatments, respectively. Another hospital-based study from France included only elderly patients aged 75 years or older. [41] In this study, where all the patients were high-risk, the proportion of patients receiving undertreatment was $58.5 \%$. Most of the remaining hospital-based studies reported guideline non-adherence based on the patients' risk of stroke. Accordingly, undertreatment in patients at high-risk of stroke ranges from $14.6 \%$ in Greece [49] to $59.1 \%$ in Italy and Spain [74]. Findings from studies incorporating multiple 
practice sites show similar rates of guideline non-adherence in high-risk patients where $15.7 \%-25.6 \%$ of patients received undertreatment. [46, 54, 64] However, the proportion of low-risk patients receiving overtreatment varied considerably. $[46,54,56,64]$ One clear observation is that undertreatment in high-risk patients attending primary healthcare is higher than for patients attending hospitals as $33.1 \%$ to $49.2 \%$ of patients were receiving undertreatment in primary healthcare. $[33,53,75]$ On the other hand, the proportion of low-risk patients receiving overtreatment appears to be lower $(0.6 \%$ to $13.1 \%)$ than their hospital counterparts. [53, 75] The above observations indicate that there may be a tendency towards not prescribing OACs in the primary healthcare setting. One multicenter study that focused on cardiology practices found the proportion of high-risk patients receiving undertreatment ranged from $11.2 \%$ to $21.7 \% \%$. [45] Close to three-quarters of low-risk patients in this study were receiving overtreatment.

\subsection{North America}

Guideline non-adherence in the U.S. and Canada also varies depending on the setting. Around $20 \%$ of patients with AF attending hospitals and the primary care setting received guideline non-adherent treatment. [38, 44, 47, 77] On the other hand, the proportion of patients receiving guideline non-adherent treatment is much higher among patients attending ambulatory settings. OAC was not provided to $39.3 \%$ to $43 \%$ patients at high risk of thromboembolism. [36, 37, 39]

\subsection{Africa and Latin America}

As compared to other parts of the world, literature on the extent of guideline non-adherence from Africa and Latin America is scarce. One single-center hospital-based Ethiopian study reported that $64.8 \%$ of patients with AF received guideline non-adherent treatment. [78] A multi-nation study from Latin America that involved multiple practices reported that $50.1 \%$ of the patients at high risk of stroke received guideline non-adherent treatment while nearly two-thirds of patients who were at low risk received over treatment. [48]

\section{Factors influencing guideline non-adherence}

Multiple factors have been associated with non-adherence to guideline recommendations and prescription of OACs. Though they may not always be evident $[56,60,64]$, the most common factors can be categorized as (a) patient-related or (b) physician-related.

\subsection{Patient-related factors}



be because of legitimate or irrational beliefs that are related to patients' knowledge. [79] Patients often feel that they are not receiving adequate information. [80] Therefore, patient resistance may be improved by providing detailed information and education. [81]

Age is one key factor for guideline non-adherence. [32, 35, 43, 61, 70, 73, 74, 82, 83] A statistically significant increase in guideline non-adherence with advancing age, mainly due to undertreatment, has been reported by multiple studies. [35, 74] This is true even after adjusting for the bleeding risk. [32, 35, 43, 70, 73, 83] While the proportion of AF patients who receive OAC decreases with advancing age $[32,35,43,70,73,74,82,83]$, this may vary depending on the age category. [73] Gundlund et al. reported that, compared to those patients younger than 65 years of age, being 65 to 74 years and $\geq 75$ years, were associated with higher and lower prescriptions of OAC after adjusting for bleeding risk, respectively. [73]

Stroke and bleeding risk or history are important predictors of guideline non-adherence and non-prescription of OACs. As one might expect, risk $[36,43,76]$ or history of $[58,83]$ stroke is directly associated with prescription of OACs, while bleeding risk [58, 70, 76] or previous bleeding [32, 35, 36, 43, 77] has an inverse association. Başaran et al. [20] reported that patients without a history of stroke were 1.4- to 1.9-times more likely to receive guideline non-adherent anticoagulation. As explained below, physicians' preferences contribute to these observations.

Because female sex was considered a risk factor for stroke, it was included in the most commonly recommended stroke risk stratification tool, the $\mathrm{CHA}_{2} \mathrm{DS}_{2}-\mathrm{VASc}$ score. However, it is clear that it has been overlooked by most clinicians as sex has emerged as one of the predictors of guideline non-adherence and non-prescription of anticoagulants in different studies, where females have higher chances of undertreatment. [35, 39, 55, 61, 73] However, the female sex category is no longer considered a significant risk for stroke, and as such, has been excluded from the $\mathrm{CHA}_{2} \mathrm{DS}_{2}$-VASc score by recent AF guidelines. [5] Though there is no clear explanation, one study discussed that prescribers' or females patients' tendencies to decline OACs could be the potential reasons for the higher rates of guideline non-adherence in female patients with AF. [39] The presence of comorbidities such as ischemic heart disease, cancer, chronic kidney disease, chronic obstructive pulmonary disease, dementia, peripheral arterial disease, and heart failure have all been associated with guideline non-adherence, in particular less OAC prescribing. [20, 32, 40, 58, 69, 73, 74] On the other hand, patients with deep venous thrombosis, 
301

302

303

304

305

306

307

308

309

310

311

312

313

314

315

316

317

318

319

320

321

322

323

324

325

326

327

328

329

hypertension, valvular heart disease, and higher body mass index are more likely to receive OACs. [20, 61, 73, 83] Prescription of antiplatelet agents or non-steroidal anti-inflammatory drugs [36, 41, 56, 64], recurrent falls or history of falls [32, 77], first detected and/or paroxysmal AF [32, 35], alcohol abuse [47, 73], pharmacological (vs electrical) cardioversion [35], rural residence and illiteracy [20], difficult access to monitoring [40] and frailty [84] have all been associated with underprescription of OACs. Most of the above factors that contribute to guideline non-adherence share the common feature of increasing the risk of bleeding and could be the underlying reason for their contribution in guideline non-adherence. One Australian study also reported that indigenous people are less likely to receive guideline-adherent treatment. [31]

\subsection{Physician-related factors}

Physician preferences make a significant contribution to guideline non-adherence, particularly those related to their beliefs and practice patterns. $[64,68,85]$ Prescribers usually focus on the risk of bleeding associated with OACs rather than the risk associated with not prescribing anticoagulants. $[20,35,54,58,70,76,80,85]$ Reports from qualitative studies indicate that prescribers use formal stroke risk assessment tools but few use formal bleeding assessment tools. [79, 86] Instead, prescribers rely on informal and subjective bleeding risk assessments. These include comorbidities, history of falls and bleeding, and age. [79, 86, 87] However, formal bleeding assessment tools such as HAS-BLED already consider comorbidities, prior history of bleeding, and age. [88] There may be a need to reassure physicians using findings such as that from a European registry-based study that indicated that the risk of stroke increases more than the risk of bleeding with advancing age, [89] and the fact that the risk of falls should not be the sole reason for withholding OACs. [16] According to a mixed-methods European study, the majority of prescribers including primary care or family physicians, cardiologists, and neurologists reported a need for skills improvement in interpreting risk stratification tools such as $\mathrm{CHA}_{2} \mathrm{DS}_{2}-\mathrm{VASc}$ and HASBLED. [21] Indeed, van Doorn et al. reported that the reason given by a quarter of GPs for overtreatment was that the responsibility of stroke prevention is the cardiologists', not theirs. [60]

\section{Conclusion}

The extent of guideline non-adherence is highly variable depending on the geographic region, the healthcare setting, the risk stratification used, and other factors. There has been a gradual improvement in guideline adherence over recent years, but non-adherence to guideline recommendations, especially undertreatment, is still a major concern in many areas. Hence, quality improvement initiatives that aim to address guideline non-adherence should be primarily focused on undertreatment. A range of patient- and physician-related factors affect guideline 
adherence. Patient-related factors include refusals, bleeding risk and recurrent falls, which are potentially modifiable, as well as non-modifiable factors such as older age, stroke risk and female sex. Clear guidance on thromboprophylaxis among elderly patients seems to be needed. A greater emphasis on the risk of bleeding than the risk of stroke among physicians also significantly contributed to guideline non-adherence. Research into how to best incorporate stroke and bleeding risk assessment tools into the workflow of physicians, improve patient knowledge and understanding of the risks and benefits of OACs, better integrate primary healthcare with higher healthcare settings and increase the involvement of allied health professionals may help minimize guideline nonadherent $\mathrm{OAC}$ treatment in patients with $\mathrm{AF}$ and thereby improve patient outcomes.

\section{Declarations}

Funding: Not applicable.

Conflict of interest: The authors declare no conflicts of interest.

Availability of data and material: All data and material used for synthesis of this review are included in this manuscript.

Code availability: Not applicable.

Ethics approval: Not applicable.

Consent to participate: Not applicable.

Consent for publication: Not applicable.

Author contributions: EAG contributed to the concept and design, and the literature search; wrote the first draft of the manuscript; critically revised the manuscript; and gave final approval of the submitted manuscript. SMS, LC, LB, and KL contributed to the concept and design; critically revised the manuscript; and gave final approval of the submitted manuscript.

\section{References}

1. Lippi G, Sanchis-Gomar F, Cervellin G. Global epidemiology of atrial fibrillation: An increasing epidemic and public health challenge. Int J Stroke. 2020:1747493019897870.

2. Pistoia F, Sacco S, Tiseo C, Degan D, Ornello R, Carolei A. The epidemiology of atrial fibrillation and 3. Goralnick E, Bontempo LJ. Atrial Fibrillation. Emerg Med Clin North Am. 2015;33(3):597-612. 

epidemic in the 21st century. Int J Cardiol. 2013;167(5):1807-24. Australia and the Cardiac Society of Australia and New Zealand: Australian clinical guidelines for the diagnosis and management of atrial fibrillation 2018. Heart Lung Circ. 2018;27(10):1209-66. thromboembolism in atrial fibrillation: a meta-analysis of trials of anticoagulants and antiplatelet drugs. J Gen Intern Med. 2000;15(1):56-67. Rhythm Association Practical Guide on the use of non-vitamin K antagonist oral anticoagulants in patients with atrial fibrillation. Eur Heart J. 2018;39(16):1330-93.

8. Mueck W, Schwers S, Stampfuss J. Rivaroxaban and other novel oral anticoagulants: pharmacokinetics in healthy subjects, specific patient populations and relevance of coagulation monitoring. Thromb J. 2013;11(1):10.

9. Alamneh EA, Chalmers L, Bereznicki LR. The Tasmanian atrial fibrillation study: Transition to direct oral anticoagulants 2011-2015. Cardiovasc Ther. 2017;35(3). focused update of the 2014 AHA/ACC/HRS guideline for the management of patients with atrial fibrillation: a report of the American College of Cardiology/American Heart Association task force on clinical practice guidelines and the Heart Rhythm Society in collaboration with the Society of Thoracic Surgeons. Circulation. 2019;140(2):e125-e51. 2018;34(11):1371-92. atrial fibrillation: CHEST guideline and expert panel report. Chest. 2018;154(5):1121-201. 

management. Korean Circ J. 2018;48(12):1033-80. management of atrial fibrillation developed in collaboration with EACTS. Eur Heart J. 2016;37(38):2893-962. (JCS 2013). Circulation Journal. 2014:CJ-66. [Available from: www.nice.org.uk/guidance/cg180. valvular atrial fibrillation? Arch Cardiovasc Dis. 2015;108(10):530-9. 18. Tomlin AM, Lloyd HS, Tilyard MW. Atrial fibrillation in New Zealand primary care: Prevalence, risk factors for stroke and the management of thromboembolic risk. Eur J Prev Cardiol. 2017;24(3):311-9. management in patients with atrial fibrillation undergoing coronary stent implantation: What is the impact of guideline adherence? Int J Cardiol. 2016;203:987-94. for stroke prevention in atrial fibrillation in different health care settings: Results from RAMSES study. Eur J Intern Med. 2017;40:50-5. system barriers to guideline implementation among European physicians treating patients with atrial fibrillation: a European Society of Cardiology international educational needs assessment. Europace. 2018;20(12):1919-28. incidence in atrial fibrillation: insights from a large German claims database. Clin Res Cardiol. 2019;108(9):104252. and atrial fibrillation guidelines in practice: barriers to and strategies for optimal implementation. Europace. 

in antithrombotic treatment for atrial fibrillation: Insights from the GLORIA-AF phase II registry. Thromb Haemost. 2017;117(12):2376-88. treatment patterns in patients with newly diagnosed nonvalvular atrial fibrillation: The GLORIA-AF Registry, Phase II. Am J Med. 2015;128(12):1306-13.e1. 26. Miyazawa K, Ogawa H, Mazurek M, Shantsila E, Lane DA, Wolff A, et al. Guideline-adherent treatment for stroke and death in atrial fibrillation patients from UK and Japanese AF registries. Circ J. 2019;83(12):243442.

27. Vallakati A, Lewis WR. Underuse of anticoagulation in patients with atrial fibrillation. Postgrad Med. 2016;128(2):191-200.

28. Wan D, Healey JS, Simpson CS. The guideline-policy gap in direct-acting oral anticoagulants usage in atrial fibrillation: evidence, practice, and public policy considerations. Can J Cardiol. 2018;34(11):1412-25.

29. Lowres N, Giskes K, Hespe C, Freedman B. Reducing stroke risk in atrial fibrillation: adherence to guidelines has improved, but patient persistence with anticoagulant therapy remains suboptimal. Korean Circulation Journal. 2019;49(10):883-907.

30. Balaghi-Inalou M, Parsa SA, Gachkar L, Andalib S. Anticoagulant Therapy in Atrial Fibrillation for Stroke Prevention: Assessment of Agreement Between Clinicians' Decision and CHA2DS2-VASc and HASBLED Scores. High Blood Press Cardiovasc Prev. 2018;25(1):61-4. anticoagulation for atrial fibrillation: a study in indigenous and non-indigenous Australians. Int J Cardiol. 2015;191:20-4. individuals with atrial fibrillation in a nursing home setting in France: comparisons of resident characteristics and physician attitude. J Am Geriatr Soc. 2015;63(1):71-6. 

stroke prevention in atrial fibrillation within the UK between 2012 and 2016: a cross-sectional analysis study using CPRD. BMJ Open. 2017;7(9):e015363.

34. Lee SR, Choi EK, Han KD, Cha MJ, Oh S, Lip GYH. Temporal trends of antithrombotic therapy for stroke prevention in Korean patients with non-valvular atrial fibrillation in the era of non-vitamin $\mathrm{K}$ antagonist oral anticoagulants: A nationwide population-based study. PLoS One. 2017;12(12):e0189495.

35. Lip GY, Laroche C, Popescu MI, Rasmussen LH, Vitali-Serdoz L, Dan GA, et al. Improved outcomes with European Society of Cardiology guideline-adherent antithrombotic treatment in high-risk patients with atrial fibrillation: a report from the EORP-AF General Pilot Registry. Europace. 2015;17(12):1777-86. non-prescription in patients with atrial fibrillation and elevated stroke risk. Am Heart J. 2018;200:24-31. anticoagulants on rates of oral anticoagulation for atrial fibrillation. J Am Coll Cardiol. 2017;69(20):2475-84.

447 38. Piccini JP, Xu H, Cox M, Matsouaka RA, Fonarow GC, Butler J, et al. Adherence to guideline-directed stroke prevention therapy for atrial fibrillation is achievable. Circulation. 2019;139(12):1497-506. the use of oral anticoagulants for atrial fibrillation: a report from the National Cardiovascular Data Registry (NCDR((R))) PINNACLE Registry. J Am Heart Assoc. 2017;6(7). octogenarian patients with atrial fibrillation at risk of stroke? Semergen. 2020. and inappropriate prescription of antiplatelet therapy in older inpatients with atrial fibrillation. Drugs Aging. 2017;34(9):701-10. of New Guidelines (RAFTING). J Cardiovasc Med (Hagerstown). 2017;18(7):545-9. 

in patients with atrial fibrillation. Am J Cardiol. 2017;120(7):1133-8. and concordance with the American Heart Association/American College of Cardiology/Heart Rhythm Society Guidelines: Findings from ORBIT-AF (Outcomes Registry for Better Informed Treatment of Atrial Fibrillation). Circ Arrhythm Electrophysiol. 2017;10(11). prevention strategies in 11096 European patients with atrial fibrillation: a report from the EURObservational Research Programme on Atrial Fibrillation (EORP-AF) Long-Term General Registry. Europace. 2018;20(5):74757. 46. Cools F, Wollaert B, Vervoort G, Verstraete S, Voet J, Hermans K, et al. Treatment patterns in anticoagulant therapy in patients with newly diagnosed atrial fibrillation in Belgium: results from the GARFIELDAF registry. Acta Cardiol. 2019;74(4):309-18.

47. Dupree L, DeLosSantos M, Smotherman C. Evaluation of adherence to guideline-directed antithrombotic therapy for atrial fibrillation at hospital discharge. J Cardiovasc Pharmacol Ther. 2018;23(6):502-8. 48. Jerjes-Sanchez C, Corbalan R, Barretto ACP, Luciardi HL, Allu J, Illingworth L, et al. Stroke prevention in patients from Latin American countries with non-valvular atrial fibrillation: Insights from the GARFIELD-AF registry. Clin Cardiol. 2019;42(5):553-60.

49. Kartas A, Samaras A, Vasdeki D, Dividis G, Fotos G, Paschou E, et al. Flaws in anticoagulation strategies in patients with atrial fibrillation at hospital discharge. J Cardiovasc Pharmacol Ther. 2019;24(3):225-32. Risk profiles and pattern of antithrombotic use in patients with non-valvular atrial fibrillation in Thailand: a multicenter study. BMC Cardiovasc Disord. 2018;18(1):174. 

affect anticoagulant use for stroke prevention in nonvalvular atrial fibrillation? The prospective global anticoagulant registry in the FIELD-Atrial Fibrillation. Circ Cardiovasc Qual Outcomes. 2015;8(2 Suppl 1):S1220.

53. Mochalina N, Joud A, Carlsson M, Sandberg MEC, Sjalander A, Juhlin T, et al. Antithrombotic therapy in patients with non-valvular atrial fibrillation in Southern Sweden: A population-based cohort study. Thromb Res. 2016;140:94-9. fibrillation and 'real world' adherence to guidelines in the Balkan Region: The BALKAN-AF Survey. Sci Rep. 2016;6:20432.

55. Sabouret P, Bricard M, Hermann MA, Cotte FE, Deret-Bixio L, Rushton-Smith S. Discrepancy between guidelines for stroke prevention in atrial fibrillation and practice patterns in primary care. The nationwide French AFIGP survey. Arch Cardiovasc Dis. 2015;108(11):544-53. anticoagulant prescription in Dutch patients with recent-onset atrial fibrillation: observations from the GARFIELD-AF registry. Thromb J. 2020;18:5.

502 57. Wertheimer G, Bereznicki LR. Exploring the quality of anticoagulant prescribed for patients with atrial 503 fibrillation at the St John of God Hawkesbury district health centre, New South Wales, Australia. J Cardiovasc 504 Pharmacol Ther. 2019;24(1):46-53.

505 58. Rathinam I, Anoopkumar-Dukie S, Badrick T, Teasdale T, Bernaitis N. Anticoagulant initiation during hospital admissions for atrial fibrillation in South-East Queensland, Australia. Heart Lung Circ. 2020. management for real-world guideline adherence: COmparison study of Drugs for symptom control and complication prEvention of Atrial Fibrillation (CODE-AF) Registry. Korean Circ J. 2017;47(6):877-87. primary care. Int J Cardiol. 2015;187:525-6. 

atrial fibrillation and a low risk of thromboembolism: Insights from the NCDR PINNACLE registry. JAMA Intern Med. 2015;175(6):1062-5. oral anticoagulation therapy in the first 3 months after the diagnosis of atrial fibrillation in Israel: A populationbased study. J Cardiovasc Electrophysiol. 2020. fibrillation-related stroke in England and its association with uptake of oral anticoagulation. Eur Heart J. 2018;39(32):2975-83. 64. Apenteng PN, Gao H, Hobbs FR, Fitzmaurice DA. Temporal trends in antithrombotic treatment of realworld UK patients with newly diagnosed atrial fibrillation: findings from the GARFIELD-AF registry. BMJ Open. 2018;8(1):e018905. valvular atrial fibrillation and anticoagulation therapy in a Japanese region- analysis using the national health insurance database. Circ J. 2020. 66. Miyazawa K, Li YG, Rashed WA, Al Mahmeed W, Shehab A, Zubaid M, et al. Secondary stroke prevention and guideline adherent antithrombotic treatment in patients with atrial fibrillation: Insights from the Gulf Survey of atrial fibrillation events (Gulf SAFE). Int J Cardiol. 2019;274:126-31. fibrillation patients. Int J Clin Pharm. 2019;41(6):1434-41.

533 68. Bista D, Chalmers L, Peterson GM, Bereznicki LRE. Patient characteristics and antithrombotic 534 prescribing patterns in patients with atrial fibrillation in Tasmania. Clin Appl Thromb Hemost. 2017;23(5):43844. 69. Aziz H, Mills D, Newbury J. Adherence to antithrombotic therapy guidelines and atrial fibrillation in a rural hospital: a clinical audit. Rural Remote Health. 2019;19(1):4342. care facilities with atrial fibrillation. Clin Appl Thromb Hemost. 2018;24(3):519-24. 
541 progression of atrial fibrillation in a nurse-led clinic. Intern Med J. 2020.

542 72. Jortveit J, Pripp AH, Langorgen J, Halvorsen S. Poor adherence to guideline recommendations among 543 patients with atrial fibrillation and acute myocardial infarction. Eur J Prev Cardiol. 2019;26(13):1373-82.

544 73. Gundlund A, Xian Y, Peterson ED, Butt JH, Gadsboll K, Bjerring Olesen J, et al. Prestroke and 545 poststroke antithrombotic therapy in patients with atrial fibrillation: Results from a nationwide cohort. JAMA $546 \quad$ Netw Open. 2018;1(1):e180171.

547 74. Proietti M, Nobili A, Raparelli V, Napoleone L, Mannucci PM, Lip GY. Adherence to antithrombotic 548 therapy guidelines improves mortality among elderly patients with atrial fibrillation: insights from the REPOSI 549 study. Clin Res Cardiol. 2016;105(11):912-20.

550 75. Mazurek M, Shantsila E, Lane DA, Wolff A, Proietti M, Lip GYH. Guideline-adherent antithrombotic treatment improves outcomes in patients with atrial fibrillation: Insights from the community-based Darlington atrial fibrillation registry. Mayo Clin Proc. 2017;92(8):1203-13.

553 76. Navarro-Juan M, Carbonell-Torregrosa MA, Palazon-Bru A, Martinez-Diaz AM, Gil-Guillen VF. 554 Nonadherence to guidelines for prescribing antiplatelet/anticoagulant therapy in patients with atrial fibrillation. Fam Pract. 2016;33(3):290-5. strategies in North American patients with atrial fibrillation: The GLORIA-AF registry program. Clin Cardiol. 2018;41(6):744-51. undertreatment in patients with atrial fibrillation attending Gondar University Hospital: a retrospective cohort study. Thromb J. 2018;16:22. study. Journal of atrial fibrillation. 2016;9(1). Patients' and physicians' perceptions and attitudes about oral anticoagulation and atrial fibrillation: a qualitative systematic review. BMC Fam Pract. 2017;18(1):3. 

anticoagulation for atrial fibrillation: interviews with clinicians. J Multidiscip Healthc. 2012;5:129.

569 82. Miller RJH, Chew DS, Rezazadeh S, Klassen S, Pournazari P, Lang E, et al. Factors influencing oral 570 anticoagulation prescription for patients presenting to emergency departments with atrial fibrillation and flutter. 571 Can J Cardiol. 2018;34(6):804-7.

572 83. Pennlert J, Asplund K, Carlberg B, Wiklund PG, Wisten A, Asberg S, et al. Antithrombotic treatment 573 following intracerebral hemorrhage in patients with and without atrial fibrillation. Stroke. 2015;46(8):2094-9.

574 84. Oqab Z, Pournazari P, Sheldon RS. What is the impact of frailty on prescription of anticoagulation in 575 elderly patients with atrial fibrillation? a systematic review and meta-analysis. J Atr Fibrillation. 2018;10(6):1870.

576 85. Kea B, Alligood T, Robinson C, Livingston J, Sun BC. Stroke prophylaxis for atrial fibrillation? To 577 prescribe or not to prescribe-a qualitative study on the decision making process of emergency department 578 providers. Ann Emerg Med. 2019;74(6):759-71.

579 86. Pokorney SD, Bloom D, Granger CB, Thomas KL, Al-Khatib SM, Roettig ML, et al. Exploring patient580 provider decision-making for use of anticoagulation for stroke prevention in atrial fibrillation: Results of the 581 INFORM-AF study. Eur J Cardiovasc Nurs. 2019;18(4):280-8.

582 87. Berti D, Moors E, Moons P, Heidbuchel H. Prevalence and antithrombotic management of atrial 583 fibrillation in hospitalised patients. Heart. 2015;101(11):884-93.

584 88. Pisters R, Lane DA, Nieuwlaat R, de Vos CB, Crijns HJ, Lip GY. A novel user-friendly score (HAS585 BLED) to assess 1-year risk of major bleeding in patients with atrial fibrillation: the Euro Heart Survey. Chest. $586 \quad 2010 ; 138(5): 1093-100$.

587 89. Patti G, Lucerna M, Pecen L, Siller-Matula JM, Cavallari I, Kirchhof P, et al. Thromboembolic risk, 588 bleeding outcomes and effect of different antithrombotic strategies in very elderly patients with atrial fibrillation: 589 a sub-analysis from the PREFER in AF (PREvention oF Thromboembolic Events-European Registry in Atrial 590 Fibrillation). J Am Heart Assoc. 2017;6(7). 